\title{
Urethral squamous cell papilloma: Case report and literature review
}

\author{
Paul Gustafson, MD, FRCSC; Howard N. Fenster, MD, FRCSC; Alan I. So, MD, FRCSC
}

The Vancouver Prostate Centre, Department of Urologic Sciences, University of British Columbia, Vancouver, BC

Cite as: Can Urol Assoc J 2014;8(5-6):e364-5. http://dx.doi.org/10.5489/cuaj.1582 Published online May 21, 2014.

\begin{abstract}
Lesions around the urethral meatus in women are common. The differential diagnosis for these lesions is extensive and includes urethral caruncle, urethral prolapse, urethral diverticulum, periurethral gland abscesses and, of most concern, urethral carcinoma. Non-invasive squamous lesions of the urethra are rare and their clinical significance is uncertain. We present a case of a urethral squamous papilloma at the urethral meatus in a premenopausal woman causing significant symptoms.
\end{abstract}

\section{Introduction}

Lesions around the urethral meatus in women are common. The differential diagnosis for these lesions is extensive and includes urethral caruncle, urethral prolapse, urethral diverticulum, periurethral gland abscesses and, of most concern, urethral carcinoma. A definitive diagnosis can unusually be reached preoperatively through a careful history, physical examination, and the use of adjunctive tests where necessary. ${ }^{1}$ The incidence of these lesions changes as women age. The hypo-estrogenic state of postmenopausal women, for instance, predisposes them to developing urethral caruncles. ${ }^{2}$ The incidence of urethral carcinoma, although rare, also increases with age, most commonly in the fifth and sixth decades of life. ${ }^{1}$ Non-invasive squamous lesions of the urethra are rare and their clinical significance is uncertain. We present a case of a urethral squamous papilloma at the urethral meatus in a premenopausal woman causing significant symptoms.

\section{Case report}

A 43-year-old female presented with a history of several months of dysuria and intermittent gross hematuria. She also described progressive obstructive symptoms, such as straining to void, poor urinary flow, and incomplete emptying in the weeks prior to the urologic consultation. She was otherwise healthy, only taking a selective serotonin reuptake inhibitor (SSRI) for depression and her only previous surgery was a Cesarean section. There were no identifiable risk factors for urothelial cell carcinoma, including no smoking history or occupational exposures to urothelial carcinogens. Her sexual history revealed that she had minimal risk factors for genital human papillomavirus (HPV) infection.

Physical examination revealed a $5-\mathrm{mm}$ erythematous, polypoid lesion emanating from the 5 o'clock position of the urethral meatus. The lesion was friable and clearly the source of bleeding. There were no visible genital warts. The remainder of the genitourinary exam was unremarkable. Cystoscopic examination revealed that the mass extended into the urethra for only a few millimeters and the remainder of the urethra appeared to be normal as was the bladder. There were no other erythematous changes or lesions noted in either the bladder or urethra.

An excisional biopsy was performed under general anesthesia without complication and the specimen was sent for pathology. Pathology revealed urethral squamous papilloma (Fig. 1).

The patient is well at last follow-up, 9 months post-resection with resolution of her irritative symptoms and hematuria and no evidence of tumour recurrence.

\section{Discussion}

There are very few documented cases of squamous papilloma in the bladder or urethra. These recorded cases have most commonly been reported in women in their 40s to 70s. The most common presenting complaint is irritative voiding symptoms. ${ }^{3}$ Given the rarity of these lesions, it is difficult to identify risk factors with confidence, but it is believed that they are similar to those associated with urothelial cell carcinoma, including smoking and exposure to aromatic amines. ${ }^{4}$ The typical visual appearance of these 


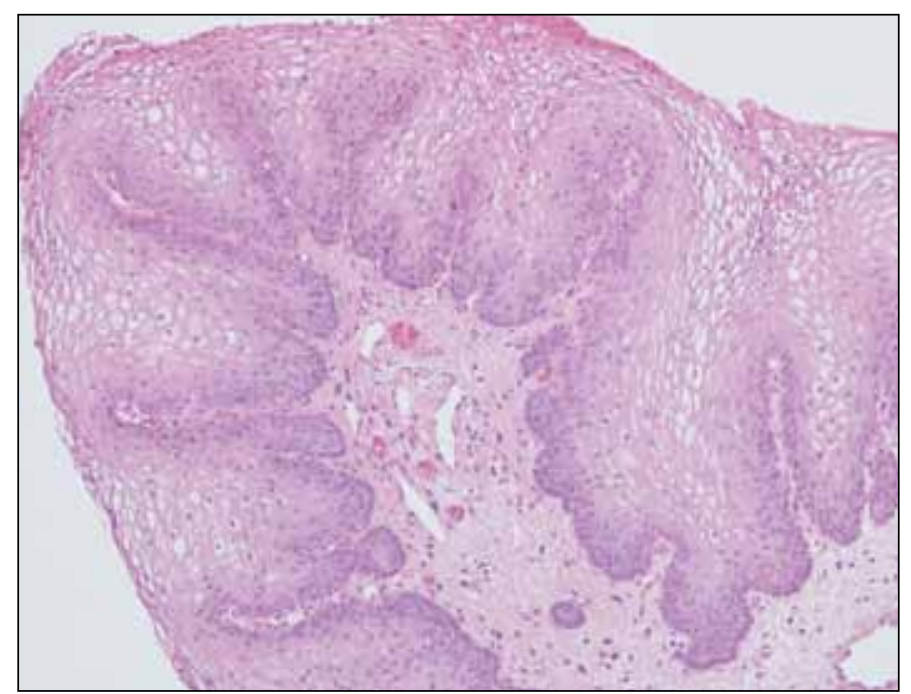

Fig. 1. Urethral squamous papilloma (p16 immunostain negative for human papillomavirus).

lesions resembles that of low-grade papillary urothelial cell carcinoma. Histologic analysis reveals a lesion with a fibrovascular core-lined with squamous cells. The squamous cells have koilocytotic changes or atypical features. ${ }^{3} \mathrm{~A}$ study by Cheng and colleagues compared these lesions to verrucous carcinoma and condylomata accuminata, 2 other proliferative squamous lesions. This study found no link between urethral squamous papilloma and HPV infection, which is in contrast to condyloma accuminata. In addition, there is typically minimal or no accumulation of the tumour suppressor protein $\mathrm{p} 53$, which is distinct from the findings in verrucous carcinoma. ${ }^{5}$

The most important differential diagnosis is to rule out urethral carcinoma. This can be accomplished through pathology review from excisional biopsy of urethral lesions to the transurethral resection of bladder tumours. The diagnosis of squamous papilloma relies on an accurate pathological review. To confirm the diagnosis, a p16 immunostain may be helpful to rule out HPV. Squamous papilloma is considered benign. Recurrence is believed to be rare following surgical removal, but the data is limited due to the small number of reported cases. ${ }^{4}$ The largest review was of 5 patients with squamous papilloma. ${ }^{6}$ Of these 5 patients, 2 were free of recurrence after an average of 15 months of follow-up. Two patients were lost to follow-up and 1 patient underwent a cystectomy after 21 months of follow-up for urothelial cell carcinoma, but this patient been diagnosed with low-grade urothelial cell carcinoma prior to the dis- covery of squamous cell papilloma. ${ }^{6}$ Our literature review revealed no case series examining squamous papilloma of the urethra; therefore recommendations of follow-up are difficult to construct. However, periodic reassessment with an urologist or primary care physician, with repeated investigations for a recurrence of symptoms, is a reasonable follow-up strategy.

This case emphasizes the importance of an accurate pathological review to the practicing urologist. Fortunately, this rare lesion was recognized and it had a benign course. A close urologist-pathologist relationship had a strong impact on the determination of positive post-urethrectomy surgical margins and the depth of invasion for bladder tumours.

\section{Conclusion}

Urethral squamous papilloma is rare and has minimal malignant potential. It presents most commonly with irritative voiding symptoms and can be managed effectively with excisional biopsy. More sinister diagnoses on the differential of a urethral tumour can be ruled out by pathological review.

Competing interests: Dr. Gustafson and Dr. Fenster declare no competing financial or personal interests. Dr. So is a member of the Speakers bureau for Amgen, Astellas and Janssen.

This paper has been peer-reviewed.

\section{References}

1. Wein AJ, MD, Kavoussi LR, Novick AC, et al. Campbell-Walsh Urology. 10th ed. Philadelphia: PA. Elselvier; 2012.

2. Conces MR, Williamson SR, Montironi R, et al. Urethral caruncle: Clinicopathologic features of 41 cases. Hum Pathol 2012;43:1400-4. http://dx.doi.org/10.1016/i.humpath.2011.10.015

3. Bostwick D, Cheng L. Urologic surgical pathology. 2nd ed. Philadelphia: PA. Elselvier; 2008.

4. Cheng L, Leibovich $B C$, Cheville JC, et al. Squamous papilloma of the urinary tract is unrelated to condyloma acuminata. Cancer 2000;88:1679-86. http://dx.doi.org/10.1002/(SICI) 1097 0142(20000401)88:7<1679::AID-CNCR23>3.0.C0;2-K

5. Lopez-Beltran A, Requena MJ, Alvarez-Kindelan J, et al. Squamous differentiation in primary urothelial carcinoma of the urinary tract as seen by MAC387 immunohistochemistry. J Clin Pathol 2007;60:332-5. http://dx.doi.org/10.1136/icp.2006.038802

6. Guo CC, Fine SW, Epstein Jl. Noninvasive squamous lesions in the urinary bladder: A clinicopathologic analysis of 29 cases. Am J Surg Pathol 2006;30:883-91.

Correspondence: Dr. Alan So, Level 6, 2775-Laure St, DHCC, Vancouver, BC V5Z 1M9; alan.so@ubc.ca 